\title{
How much weight should viral load carry?
}

It was a strange meeting, even for an AIDS-related advisory committee to the US Food and Drug Administration (FDA). Gathering for what was billed as a hearing to recommend approval of Roche's PCR-based HIV viral load assay (see Nature Medicine 1, 980; 1995), the members of the joint Blood Products/Antiviral Drug advisory committee instead heard a plea for help. FDA officials, who want to approve the test as soon as possible, asked committee members to help them write the equivalent of a "package insert": How is this test best used, and what do different results mean to treatment decisions? As became clear from the advisory committee's discussion, answers to these questions are still uncertain. But, in fact, many clinicians are already using viral load measurements to manage their AIDSinfected patients (at least those that can afford to pay for the unapproved tests at $\$ 175$ to $\$ 200$ apiece). This puts the FDA in the odd position of being forced to approve an expensive test that is already in fairly wide use, without the extensive clinical data the agency normally requires as the basis for approval.

The past year has seen basic AIDS research place powerful new antiviral weapons into the hands of clinicians (the protease inhibitors - see Nature Medicine 2,$274 ; 1996$ ), although no one is sure when in the course of disease to start using them. Ironically, many believe that decision is dictated by the results of the (unapproved) viral load assay. In an attempt to clear up the confusion, several different groups of clinical researchers are either planning to issue guidelines (in Europe and Canada) or are rushing consensus statements into press (in the United States) on the use of the assay in making treatment decisions. As these reports are not yet available, Nature Medicine asked leading AIDS clinical researchers how they actually use the test for managing their AIDS patients.

The "bottom line" of therapy decisions for all the clinicians contacted is to reduce viral load below detectable levels in patients using the minimum number and combination of antiviral drugs effective at doing so. To that end, most suggest obtaining two initial viral load tests, spaced one to two weeks apart, to determine a "baseline" HIV RNA level (a third test may be necessary if the two initial tests are widely divergent). The baseline number will determine, for most clinicians, whether or not to start treatment (although a few say they would begin treatment with antiretroviral drugs regardless of the viral load). Everyone believes that some kind of combination therapy (two or three nucleoside analogue reverse transcriptase inhibitors and maybe a protease inhibitor) is called for if the viral load is greater than 10,000 copies per milliliter of blood. The specific form that treatment takes is determined on an individual patient basis, depending on the person's previous history with antiviral treatment, his or her tolerance for the side effects of the different available drugs, and, in the case of newer or experimental treatments, the ability of the patient to bear the cost.

Where there is significant disagreement between clinicians is over patients with numbers in the "gray" range of 500 to 10,000 copies. The researchers are divided between those who would treat such patients aggressively (arguing that even that relatively low levels of HIV replication favors the development of strains resistant to the antiviral drugs), and those who would hold back and see if the load continued to increase (believing that the immune system is still doing a "sufficient" job of keeping viral replication in check). Regardless of their approach, all of the researchers contacted believed that the viral load should be checked every three to four months to monitor the effectiveness of the current therapy. A significant change upward (half a log or more), or no change from the baseline number after beginning therapy, would dictate either altering the dose of the current drug or, more likely, switching to another combination. They also all emphasize that the patients should play an active role in making treatment decisions.

There is no doubt that viral load is a powerful prognostic tool, predicting progression to AIDS and death. There is also no doubt it is an effective tool for assessing the immediate impact of antiviral drugs, offering the possibility of rapidly screening new candidate antiviral drugs. However, if FDA only approves the test for these purposes, many managed care and insurance companies will be unwilling to pay for tests the number of times necessary for patient management. But to approve the test for long-term patient management, FDA would be more com- fortable with data that prove that repressing viral load for longer periods of time translates into clinical benefit. Although a few prospective trials have been proposed, it is going to be difficult at best to enroll patients into trial arms that are less than state of the art.

Despite the push for widespread use, there are serious reasons to question the universal utility of the assays. Researchers have observed a significant number of "outliers" (patients who have both high $\mathrm{CD} 4^{+} \mathrm{T}$-cell counts and high viral load, or low CD4 and low viral load); some patients with high CD4 and low viral load still clinically decline: it's not clear that viral levels in the plasma (which the viral load assay measures) correlate with viral levels in other im. munological "compartments" (such as lymph nodes). Unexplained observations like these make FDA edgy, and rightly so, about approving the test for patient management.

But edginess isn't a sufficient reason for only partially approving an important tool. The optimal use of the test will become clear as more patients and physicians have access to it. All the researchers interviewed said FDA should approve the assay for use as soon as possible, and should emphasize in the package insert the only universal condition demanded by the advisory committee: physician education as to the meaning and current limits of the assay's results.

Fintan R. STEELE

\section{DID YOU KNOW?}

\section{Medical professionals top Japanese list of tax cheaters} Tokyo's private hospital operator-owners and medical practitioners not only looked after their patients' interests last year, they also headed the Tokyo Tax Authorities' list of individuals who misreported their income when filing their annual tax returns.

In fact, medical professionals dominated the top ten list, including physicians (ranked sixth), ophthalmologists (seventh) and surgeons (eighth).

RICHARD NATHAN Tokyo, Japan 